\title{
ON STARSHAPED REARRANGEMENT AND APPLICATIONS ${ }^{1}$
}

\author{
BERNHARD KAWOHL
}

\begin{abstract}
A radial symmetrization technique is investigated and new properties are proven. The method transforms functions $u$ into new functions $u^{*}$ with starshaped level sets and is therefore called starshaped rearrangement. This rearrangement is in general not equimeasurable, a circumstance with some surprising consequences. The method is then applied to certain variational and free boundary problems and yields new results on the geometrical properties of solutions to these problems. In particular, the Lipschitz continuity of free boundaries can now be easily obtained in a new fashion.
\end{abstract}

Introduction. In [26] G. Szegö introduced the concept of radial symmetrization. This is a nonequimeasurable rearrangement which transforms functions $u: \Omega \rightarrow \mathbf{R}$, $\Omega \subset \mathbf{R}^{2}$ with starshaped level sets $\Omega_{c}:=\{x \in \bar{\Omega} \mid u(x) \geq c\}$ into functions $u^{*}$ with symmetric starshaped level sets. Later $[2,3,15,16,22]$ his approach was generalized to transform functions with level sets containing the origin into starshaped and not necessarily symmetric level sets and to higher dimensions. We call a set $D \subset \mathbf{R}^{n}$ starshaped with respect to $x_{0}$ if $x \in D$ implies $t x+(1-t) x_{0} \in D$ for every $t \in[0,1]$. We call a set $D$ starshaped if it is starshaped with respect to the origin. Therefore a more appropriate name for this rearrangement might be "starshaped rearrangement," in particular, since the notion of radial symmetrization can be easily confused with circular or spherical symmetrization or spherically symmetric rearrangement.

One of the standard results in the theory of rearrangements is that the capacity of a condenser decreases under rearrangement; i.e.,

$$
\int_{\Omega}|\nabla u(x)|^{p} d x \geq \int_{\Omega^{*}}\left|\nabla u^{*}(x)\right|^{p} d x \quad \text { for } p \geq 1,
$$

where $u \equiv 1$ on $\Omega_{1}, u=0$ on $\partial \Omega$ and $U_{\varepsilon}(0) \subset \Omega_{1} \subset \Omega, \varepsilon>0,0 \leq u \leq 1$ in $\Omega$.

This feature can be found for starshaped rearrangement, too $[\mathbf{2}, \mathbf{3}, \mathbf{1 5}, \mathbf{1 6}$, 22] but only at the sacrifice of defining different rearrangements $u^{*(p)}$ for different values of $p$. Corollary 1.4 states

$$
\int_{\Omega}|\nabla u(x)|^{p} \geq \int_{\Omega}\left|\nabla u^{*(p)}(x)\right|^{p} d x \quad \text { for } p \geq 1,
$$

provided $\Omega \subset \mathbf{R}^{n}$ is a bounded starshaped domain containing $U_{\varepsilon}(0)$ and $u: \bar{\Omega} \rightarrow$ $[0,1], u \in C^{0,1}(\bar{\Omega}), u \equiv 1$ in $U_{\varepsilon}(0)$ and $u=0$ on $\partial \Omega$.

Another convenient feature of other rearrangements is their equimeasurability. A rearrangement $u \rightarrow u^{*}$ is called equimeasurable if the $n$-dimensional Lebesgue

Received by the editors March 26, 1984, and, in revised form, September 24, 1985.

1980 Mathematics Subject Classification. Primary 35J20, 35R35, 35B05, 26D20.

${ }^{1}$ This research was supported by the Deutsche Forschungsgemeinschaft (DFG) and by E. Reid. 
measure $m_{n}\left(\Omega_{c}\right)$ coincides with $m_{n}\left(\Omega_{c}^{*}\right)$ for all $c \in$ range $u$, where $\Omega_{c}^{*}$ is the corresponding level set of $u^{*}$. Among other things, equimeasurability implies Cavalieri's principle

$$
\int_{\Omega} F(u(x)) d x=\int_{\Omega^{*}} F\left(u^{*}(x)\right) d x
$$

for every Borel measurable function $F$.

Unfortunately $u$ and $u^{*(p)}$ (for $p \geq 1$ ) are not equimeasurable, although $u$ and $u^{*(0)}$ are. Since property $(0.2)$ appears to be desirable for potential applications, one might ask if, in addition to (0.1), the inequality

$$
\int_{\Omega}|\nabla u(x)|^{p} d x \geq \int_{\Omega}\left|\nabla u^{*(0)}(x)\right|^{p} d x
$$

holds under the same conditions as (0.1).

As a first result we give a counterexample to (0.3). The proof is based on a surprising feature of this kind of rearrangement. Unlike Schwarz and Steiner symmetrization [23], the "surface" or perimeter of a level set can increase under rearrangement.

As a second result we discuss the strict inequality in (0.1). If $u$ is smooth in a sense defined below, the equality sign in $(0.1)$ implies $u=u^{*(p)}$. This is relevant because it has applications to variational problems with multiple solutions.

A third result is the fact that for nonequimeasurable rearrangements, equality (0.2) can be replaced by an inequality.

Therefore starshaped rearrangement can be applied to variational functionals of type

$$
J(v)=\int_{\Omega}\left\{|\nabla v(x)|^{p}+F(v)\right\} d x
$$

or of type

$$
J(v)=\int_{\Omega}\left\{|\nabla v(x)|^{2}+\chi_{\{v>0\}}\right\} d x
$$

Our results provide new statements about the geometry of level sets of solutions, e.g., Lipschitz continuity of the free boundary in a "jet problem" in $\mathbf{R}^{n}[\mathbf{1}]$. They also give new proofs of some already known results.

Other ways to prove starshapedness of level sets of a function $u$ are usually based on maximum principles and require fairly strong regularity properties of $u$, which are unrealistic for our applications. We refer to $[\mathbf{1 1 - 1 3}, \mathbf{1 9}, \mathbf{2 4}]$ for those and similar questions.

Throughout the paper we use the common notation $W^{1, p}(\Omega), W_{0}^{1, p}(\Omega)$ and $L^{p}(\Omega)$ for Sobolev spaces of functions $[\mathbf{9}, \mathbf{1 8}] . C(D)$ denotes functions that are continuous in $D$. If $D$ is closed, they are continuous on $D . C^{k, \alpha}(D)$ are functions with derivatives up to order $k$ and with $\alpha$-Hölder continuous $k$ th derivative, $0 \leq \alpha \leq 1$. A boundary $\partial \Omega$ of a domain $\Omega$ in $\mathbf{R}^{n}$ is said to be of class $C^{k, \alpha}$ if it can be described locally by a $C^{k, \alpha}$ function of $n-1$ variables. $C^{0,1}$ boundaries $\partial \Omega$ are called Lipschitz continuous. As usual $\mathbf{R}^{+}$denotes $(0, \infty), \mathbf{R}_{0}^{+}=[0, \infty), \mathbf{N}=\{1,2,3, \ldots\}$, $\mathbf{N}_{0}=\{0,1,2,3, \ldots\}$ and $\varepsilon$ and $\delta$ are sufficiently small positive real numbers. Unless otherwise indicated, all occurring integrals are understood to be Lebesgue integrals. This paper is a revised and abbreviated version of $[\mathbf{1 4}]$. 
ACknowledgment. I thank C. Bandle, J. I. Diaz, H. Grabmüller, M. Marcus and G. Talenti for various helpful discussions and correspondence on the subject of rearrangement. This work was essentially done at the Lefschetz Center for Dynamical Systems in Providence, R.I. I am gratefully indebted to C. Dafermos and J. Hale for their hospitality.

I. Starshaped rearrangement. A common principle of symmetrization or rearrangement techniques is to replace a level set $\Omega_{c}:=\{x \in \bar{\Omega} \mid u(x) \geq c\}$ by another one $\Omega_{c}^{*}$ which has the desired properties of symmetry or, in our case, starshapedness. The function $u^{*}$ can then be constructed from its level sets $\Omega_{c}^{*}$ just like a three-dimensional mountain can be constructed from a map that shows all of its level lines.

For our purposes we shall define the rearrangement $u^{*(p)}$ of a function $u$ under the following general assumptions.

$$
\Omega \subset \mathbf{R}^{n} \text { is a bounded starshaped domain containing } U_{\varepsilon}(0) .
$$

$$
u:=\bar{\Omega} \rightarrow[0,1], u \in C^{0,1}(\bar{\Omega}), u \equiv 1 \text { in } U_{\varepsilon}(0) \text { and } u=0 \text { on } \partial \Omega .
$$

Therefore each nonempty level set of $u$ will contain $U_{\varepsilon}(0)$. Let $D \subset \mathbf{R}^{n}$ be compact and $U_{\varepsilon}(0) \subset D$. To define $D^{*(p)}$, we shall use $n$-dimensional polar coordinates $\left(r, \theta_{1}, \ldots, \theta_{n-1}\right)$ of a point $x=\left(x_{1}, \ldots, x_{n}\right) \in \mathbf{R}^{n}$, which are defined by the relations

$$
\begin{aligned}
& |x|=r, \quad x_{1}=r \cos \theta_{1}, \ldots, \\
& x_{k}=r \sin \theta_{1} \sin \theta_{2} \cdots \sin \theta_{k-1} \cos \theta_{k} \quad \text { for } k=2, \ldots, n-1, \\
& x_{n}=r \sin \theta_{1} \sin \theta_{2} \cdots \sin \theta_{n-1},
\end{aligned}
$$

where $0 \leq \theta_{k} \leq \pi$ for $k=1, \ldots, n-2$ and $-\pi \leq \theta_{n-1} \leq \pi$. For typographical reasons let $\theta$ denote the vector of the angular coordinates $\left(\theta_{1}, \ldots, \theta_{n-1}\right)$ and $T$ the $(n-1)$-dimensional hypercube $[0, \pi]^{n-2} \times[-\pi, \pi]$. A point $x \in \mathbf{R}^{n}$ has the representation $x=(r, \theta)$, where $\theta \in T$ and $r \in \mathbf{R}_{0}^{+}$. The ray $\left\{x \in \mathbf{R}^{n} \mid x=\right.$ $\left.(r, \theta), \theta=\theta^{\prime}, r \geq \varepsilon\right\}$ will be denoted by $L\left(\theta^{\prime}\right)$ and $D\left(\theta^{\prime}\right)=D \cap L\left(\theta^{\prime}\right)$.

Let $g: \mathbf{R}^{+} \rightarrow \mathbf{R}$ be a positive and continuous function and let $G$ be its primitive. We define

$$
\begin{aligned}
h(\theta) & :=\int_{D(\theta)} g(r) d r+G(\varepsilon), \\
R(\theta) & :=G^{-1}(h(\theta))
\end{aligned}
$$

where the integral in (1.4) is understood to be a Lebesgue integral. Notice that $R(\theta)$ does not depend on $\varepsilon$. For compact sets $D$ containing $U_{\varepsilon}(0)$ we define

$$
D^{*}:=\left\{x \in \mathbf{R}^{n}|0 \leq| x \mid \leq R(\theta), \theta \in T\right\} .
$$

If $D$ is empty, then by definition $D^{*}$ is empty.

This construction transforms given sets $D$ into starshaped ones, since the sets $D^{*}$ are starshaped with respect to zero. In particular, let us from now on consider a special class of rearrangements, namely those induced by the family of metrics

$$
g(r)=r^{\beta-1} \quad \text { with } \beta \in \mathbf{R} .
$$


In addition to the assumption $U_{\varepsilon}(0) \subset D$ and $D$ compact, let us now assume that the rays $\theta=$ constant intersect $\partial D$ in a finite (odd) number $2 m+1$ of points

$$
0<\varepsilon \leq r_{1}(\theta)<r_{2}(\theta)<\cdots<r_{2 m+1}(\theta) \text { with } m \in \mathbf{N}_{0} \text {. }
$$

Then for $\beta \neq 0$

$$
\begin{aligned}
& h(\theta)=\frac{1}{\beta}\left(r_{1}^{\beta}-r_{2}^{\beta}+\cdots+r_{2 m+1}^{\beta}\right), \\
& R(\theta)=\left(r_{1}^{\beta}-r_{2}^{\beta}+\cdots+r_{2 m+1}^{\beta}\right)^{1 / \beta},
\end{aligned}
$$

while for $\beta=0$

$$
\begin{aligned}
h(\theta) & =\log r_{1}-\log r_{2}+\cdots+\log r_{2 m+1}, \\
R(\theta) & =\frac{r_{1} r_{3} r_{5} \cdots r_{2 m+1}}{r_{2} r_{4} \cdots r_{2 m}} .
\end{aligned}
$$

The rearrangement of $D$ under the metric $g(r)=r^{n-p-1}$ will be denoted by $D^{*(p)}$ from now on, where $p \in \mathbf{R}, n \in \mathbf{N}$. A simple calculation shows

$$
\int_{D} d x=\int_{D^{*}(0)} d x
$$

for compact sets $D$ containing $U_{\varepsilon}(0)$. Now we are in a position to define the (decreasing) starshaped rearrangement $u^{*(p)}$ of a function $u$ satisfying (1.1), (1.2) as follows:

$$
u^{*(p)}(x):=\sup \left\{c \in[0,1] \mid x \in \Omega_{c}^{*(p)}\right\} \quad \text { for } x \in \bar{\Omega}^{*}(=\bar{\Omega}) .
$$

Then it is well known $[\mathbf{2}, \mathbf{3}, \mathbf{1 5}, \mathbf{1 6}, \mathbf{2 2}]$ that for every $p \geq 1$

$$
\Omega_{c}^{*(p)}=\left\{x \in \bar{\Omega} \mid u^{*(p)}(x) \geq c\right\} \quad \text { for each } c \in[0,1]
$$

so that the level sets of $u^{*(p)}$ are starshaped and

$$
u^{*(p)} \text { is uniformly Lipschitz continuous on } \bar{\Omega} \text {. }
$$

A new result is the following.

THEOREM 1.1. There exist a domain $\Omega \subset \mathbf{R}^{2}$ and a function $v \in W_{0}^{1,1}(\Omega)$ satisfying (1.1), $(1,2)$, for which $(0,1)$ fails; i.e.,

$$
\int_{\Omega}\left|\nabla v^{*(0)}(x)\right| d x>\int_{\Omega}|\nabla v(x)| d x
$$

holds.

The proof is based on H. Grabmüller's "long nose" (Lemma 1.2) and on Federer's coarea formula.

LEMMA 1.2. There exists a compact domain $D \subset U_{\varepsilon}(0)$ in $\mathbf{R}^{2}$ such that the perimeter of $D$ is shorter than the perimeter of $D^{*(0)}$.

To prove Lemma 1.2 we simplify a construction in $[\mathbf{1 0}]$. Let $D$ be the union of an $\varepsilon$-neighborhood of zero with $0<\varepsilon \ll 1$ and of the angular sector $\{x=(r, \theta) \in$ $\left.\mathbf{R}^{2}|1 \leq r \leq 2,| \theta \mid \leq \varphi\right\}$ where $\varphi \in(0, \pi)$ is determined below. The perimeter of $D$ 
has length $|\partial D|=2+3 \varphi+2 \pi \varepsilon ; D^{*(0)}=\left\{x=(r, \theta) \in \mathbf{R}^{2}|0 \leq r \leq \sqrt{3}+O(\varepsilon),| \theta \mid \leq\right.$ $\varphi\} \cup U_{\varepsilon}(0)$ and the perimeter of $D^{*(0)}$ has length $\left|\partial D^{*(0)}\right|=2 \sqrt{3}+\sqrt{3} \varphi+O(\varepsilon)$. Apparently $\left|\partial D^{*(0)}\right|>|\partial D|$ for sufficiently small $\varphi$, which proves the lemma.

To prove Theorem 1.1 let $\Omega \subset \mathbf{R}^{2}$ be a ball with radius 3 and center in the origin and let $D$ be the set constructed in the proof of Lemma 1.2. $D$ will be the support of a function $v: \Omega \rightarrow[0,1]$ defined by

$$
v(x):= \begin{cases}0 & \text { if } x \in \bar{\Omega} \backslash D \\ 2 / \varepsilon \cdot d(x, \partial D) & \text { if } x \in D \text { and } d(x, \partial D) \leq \varepsilon / 2 \\ 1 & \text { if } x \in D \text { and } d(x, \partial D)>\varepsilon / 2\end{cases}
$$

If $\varepsilon$ and $\varphi$ are sufficiently small, we have the strict inequality $\left|\partial \Omega_{c}\right|<\left|\partial \Omega_{c}^{*(0)}\right|$ for every $c \in(0,1)$, according to Lemma 1.2. But Federer's coarea formula states [5]

$$
\int_{\Omega}|\nabla v(x)| d x=\int_{\mathbf{R}} P(t) d t
$$

where $P(t)=$ perimeter of $\{x \in \Omega \mid w(x)>t\}$. This implies (1.13).

To formulate the next theorem, we introduce some definitions. We call a function $u: \Omega \rightarrow[0,1]$ simple if $u$ satisfies $(1.1),(1.2)$ and if $u$ is piecewise linear in $\Omega$. We call a function $u: \Omega \rightarrow[0,1]$ smooth if $u$ satisfies $(1.1),(1.2)$ and if $u$ has the properties

(i) For almost every $\theta \in T$ and every $c \in(0,1)$ the set of points $\{(r, \theta) \in$ $\Omega \mid u(r, \theta)=c\}$ is finite.

(ii) For almost every $\theta \in T$ the set of points $\{(r, \theta) \in \Omega \mid(\partial u / \partial r)(r, \theta)=0$ and $u(r, \theta) \in(0,1)\}$ is finite.

THEOREM 1.3. Let $u$ be simple or smooth and $p \geq 1$ and let $H(\theta, t): T \times[0,1] \rightarrow$ $\mathbf{R}^{+}$be continuous. Then the inequality

$$
\int_{\Omega} H\left(\theta, u^{*(p)}\right)\left|\nabla u^{*(p)}(x)\right|^{p} d x \leq \int_{\Omega} H(\theta, u)|\nabla u(x)|^{p} d x
$$

holds, and for $p>1$ equality holds only if $u=u^{*(p)}$.

Inequality (1.15) was derived in $[2,3]$ under the additional assumption $H \equiv 1$. A careful inspection of their proof reveals the extension given here and, in particular, the strictness of this inequality. The reader who wants to check this should observe that in the notation of $[\mathbf{3}]$, one has to set $p(\rho)=\rho+\varepsilon^{n-p}$ for $n \neq \alpha$ and $p(\rho) \equiv 1$ for $n=\alpha$.

COROLlaRY 1.4. Let u satisfy (1.1), (1.2). Then the following holds for $p \geq 1$ :

$$
\int_{\Omega}\left|\nabla u^{*(p)}(x)\right|^{p} d x \leq \int_{\Omega}|\nabla u(x)|^{p} d x .
$$

This follows with more or less standard approximation arguments and using a trick from [6]. One approximates $u$ by simple functions $u_{n}$ and shows that $\left\{u_{n}^{*(p)}\right\}_{n \in \mathbb{N}}$ has a subsequence converging to $u^{*(p)}$ in $W_{0}^{1, p}(\Omega)$. At the suggestion of the referee the details are left to the reader.

Next we intend to find a substitute for $(0.2)$. To this end we want to relate $u^{*(0)}$ to $u^{*(p)}$ for $p \geq 1$ or $\Omega_{c}^{*(0)}$ to $\Omega_{c}^{*(p)}$. 
LEMMA 1.5. Let $m \in \mathbf{N}_{0}$ and $0<\varepsilon \leq r_{1}<r_{2}<\cdots<r_{2 m+1}, r_{j} \in \mathbf{R}$, let $2 \leq n \in \mathbf{N}$ and $1 \leq p<\infty, p \neq n$. Then the following inequalities hold:

$$
\begin{gathered}
\left(r_{1}^{n-p}-r_{2}^{n-p}+\cdots+r_{2 m+1}^{n-p}\right)^{1 /(n-p)} \leq\left(r_{1}^{n}-r_{2}^{n}+\cdots+r_{2 m+1}^{n}\right)^{1 / n} \\
\frac{r_{1} \cdot r_{3} \cdots r_{2 m+1}}{r_{2} \cdot r_{4} \cdots r_{2 m}} \leq\left(r_{1}^{n}-r_{2}^{n}+\cdots+r_{2 m+1}^{n}\right)^{1 / n}
\end{gathered}
$$
to

If we introduce the notation $y_{j}=r_{j}^{n}, \alpha=(n-p) / n$, inequality (1.17) is equivalent

$$
F\left(y_{1}, \ldots, y_{2 m+1}\right):=\left(y_{1}^{\alpha}-y_{2}^{\alpha}+\cdots+y_{2 m+1}^{\alpha}\right)^{1 / \alpha}-y_{1}+y_{2}-\cdots-y_{2 m+1} \leq 0
$$

For $n>p$ or $\alpha>0$ inequality (1.19) is recorded in [20], and the proof is reduced to Weinberger's inequality $[\mathbf{1 7}, \mathrm{p}$. 112]. For $p>n$ or $\alpha<0$ one can either modify the proof of Payne and Weinstein in an obvious fashion or apply Theorem 5 on p. 112 in $[\mathbf{1 7}]$.

Finally l'Hospital's rule implies that (1.18) follows from (1.17). This was kindly pointed out by the referee. Another proof of (1.18) would follow along the lines of [20].

THEOREM 1.6. Let $u$ satisfy (1.1), (1.2), and $p \geq 1$. Then the following inequalities hold:

$$
u^{*(0)}(x) \geq u^{*(p)}(x) \quad \text { in } \Omega
$$

and

$$
\int_{\Omega} F(u(x)) d x \geq \int_{\Omega} F\left(u^{*(p)}(x)\right) d x
$$

for $F:[0,1] \rightarrow \mathbf{R}$ monotone nondecreasing and Borel measurable.

It suffices to prove this theorem for simple functions; the rest follows from an approximation argument. The level sets of simple functions are polyhedral, so for almost every $\theta \in T$ the boundary of a level set is intersected only finitely often, an odd number of times. Now we recall (1.7), (1.8) and Lemma 1.5 to see that

$$
\Omega_{c}^{*(0)} \supset \Omega_{c}^{*(p)} \text { modulo a nullset, }
$$

so that $(1.20)$ holds a.e. in $\Omega$, and by continuity of $u^{*(0)}$ and $u^{*(p)}(1.20)$ holds everywhere in $\Omega$. Now (1.21) follows from (1.20), (1.9) and (0.2), since

$$
\int_{\Omega} F(u(x)) d x=\int_{\Omega} F\left(u^{*(0)}(x)\right) d x \geq \int_{\Omega} F\left(u^{*(p)}(x)\right) d x
$$

To conclude our results on starshaped rearrangement, we give a counterexample to another type of inequality,

$$
\int_{\Omega} u(x) v(x) d x \leq \int_{\Omega} u^{*}(x) v^{*}(x) d x
$$

which does hold for Schwarz, Steiner or any other equimeasurable rearrangement. 
COROLLARY 1.7. There exist a domain $\Omega \subset \mathbf{R}^{2}$ and functions $u$ and $v$ satisfying (1.1), (1.2) such that for $p \geq 1$ inequality (1.23) fails. Moreover, for these functions even the following inequality holds:

$$
\int_{\Omega} u^{*(p)}(x) v(x) d x>\int_{\Omega} u^{*(p)}(x) v^{*(p)}(x) d x .
$$

To prove this corollary we extend the definition of starshaped rearrangement to characteristic functions of compact sets containing $U_{\varepsilon}(0)$. Then we set $\tilde{u}(x)=$ $\tilde{u}^{*(p)}(x)=\chi_{B_{3(0)}}(x)$, where $B_{3}(0)$ is the ball with radius 3 and center in the origin. We set $\tilde{v}(x)=\chi_{R}(x)$ where $R=B_{\varepsilon}(0) \cup\left(B_{2}(0) \backslash B_{1}(0)\right)$ and verify

$$
\int_{\Omega} \tilde{u}^{*(p)}(x) \tilde{v}(x) d x \geq \int_{\Omega} u^{*(p)}(x) \tilde{v}^{*(p)}(x) d x
$$

by computation, using (1.7) and (1.8). Now (1.24) follows after mollification of $\tilde{v}$ and $\tilde{u}$.

\section{Applications.}

EXAMPLE 1. Capacitory problems. Let $\Omega_{1} \subset \mathbf{R}^{n}$ be starshaped with respect to the origin and contain $U_{\varepsilon}(0)$. Let $\Omega_{1} \Subset \Omega_{0} \subset \mathbf{R}^{n}$, where $\Omega_{0}$ is bounded and starshaped with respect to zero. Let $u \in C^{0,1}\left(\bar{\Omega}_{0}\right), 0 \leq u \leq 1$, be a solution to the variational problem, $p \geq 1$,

(V1) $\operatorname{Min}_{v \in A_{1}} J_{1}(v):=\int_{\Omega}\left\{\frac{1}{p}|v(x)|^{p}+F(v(x))\right\} d x$,

$$
\text { where } A_{1}:=\left\{v \in W_{0}^{1, p}\left(\Omega_{0}\right) \mid v \equiv 1 \text { on } \Omega_{1}\right\} \text {. }
$$

COROllaRY 2.1. Suppose $F:[0,1] \rightarrow \mathbf{R}$ is monotone nondecreasing and continuous and that at least one of the conditions (i), (ii) or (iii) holds:

(i) Problem (V1) has a unique solution.

(ii) Problem (V1) has only smooth solutions.

(iii) If the problem has two different solutions $u$ and $w$, then either $\operatorname{supp} u \supset$ $\operatorname{supp} w$ and $u>w$ in $\operatorname{supp} u \backslash \bar{\Omega}_{1}$, or $\operatorname{supp} w \supset \operatorname{supp} u$ and $w>u$ in supp $w \backslash \bar{\Omega}_{1}$. Furthermore, let $F$ be nonconstant.

Then every solution $u$ of problem (V1) has starshaped level sets.

Under assumptions (i) or (ii) the proof follows from Theorem 1.3, Theorem 1.6 and from the observation $J_{1}\left(u^{*(p)}\right)=J_{1}(u)$. Under assumption (iii) we note that if $u$ is a solution, then so is $u^{*(p)}$ and set $w=u^{*(p)}$ to reach a contradiction. Therefore, $u=u^{*(p)}$.

If $F$ is differentiable with derivative $f:[0,1] \rightarrow \mathbf{R}$, then solutions $u$ of the variational problem (V1) are weak solutions of the degenerate elliptic boundary value problem

$$
\begin{gathered}
\operatorname{div}\left(|\nabla u|^{p-2} \nabla u\right)=f(u) \quad \text { in } \Omega, \\
u=1 \quad \text { on } \partial \Omega_{1}, \quad u=0 \text { on } \partial \Omega_{0} .
\end{gathered}
$$

If $F$ is convex with subdifferential $f=\partial F$, then $u$ is the unique weak solution of the differential inclusion

$$
\operatorname{div}\left(|\nabla u|^{p-2} \nabla u\right) \in f(u) \text { in } \Omega
$$

under boundary conditions (2.2). 
Previous versions of Corollary 2.1 for special $p$ and $F$ (mainly $F \equiv 0, p=2$ ) are known $[2,3,11,13,15,16,22]$. The novelty is that the starshapedness can now be derived without monotonicity assumptions on $f$ and without differentiability assumptions on $f$ or $u$.

Finally we want to indicate a case in which assumption (iii) of Corollary 2.1 is satisfied. In the paper of A. Friedman and D. Phillips [9] one can find that (iii) holds, provided

$$
p=2, \quad f(t)=\left\{\begin{array}{ll}
t^{\alpha} f_{0}(t) & \text { for } t \geq 0 \\
0 & \text { for } t<0
\end{array} \text { for some } \alpha \in(0,1)\right.
$$

and $m \leq f_{0}(t) \leq M, 0<m \leq M<\infty$.

EXAMPLE 2. Exterior free boundary problems in the context of reaction-diffusion. Let $\Omega_{1}$ be given as before and let $u: \mathbf{R}^{n} \rightarrow[0,1]$ be a Lipschitz continuous solution of the variational problem

$$
\begin{aligned}
\operatorname{Min}_{v \in A_{2}} J_{2}(v):=\int_{\Omega}\left\{\frac{1}{p}|\nabla v(x)|^{p} d x+\lambda^{2} F(v(x))\right\} d x \\
\text { where } A_{2}:=\left\{v \in W_{\text {loc }}^{\mathbf{1 , 2}}\left(\mathbf{R}^{n}\right) \mid v \equiv 1 \text { on } \bar{\Omega}_{1}\right\}
\end{aligned}
$$

where $F: \mathbf{R} \rightarrow \mathbf{R}_{0}^{+}$is a convex, monotone nondecreasing function with $F(0)=0$, and where $\lambda \in \mathbf{R}^{+}$is given. Furthermore, suppose that the support of $u$ is bounded.

A sufficient condition for $(2.5)$ is

$$
\int_{0}^{1}[F(t)]^{-1 / p} d t<\infty
$$

as was kindly pointed out to me by J. I. Diaz [4]. As $\lambda$ tends to infinity, the support of $u$ shrinks to a boundary layer; see $[7,9]$. As a consequence of Corollary 2.1 the support of $u$ and all level sets will be starshaped with respect to zero. A further consequence is

COROllaRY 2.2. Suppose, in addition to the above assumptions on $\Omega_{1}$ and $F$, that

$$
\begin{aligned}
& \Omega_{1} \text { is starshaped with respect to each point } \\
& y \text { in a small nonempty closed neighborhood } \\
& U_{\delta}(0) \text { of the origin. }
\end{aligned}
$$

Then all the level sets $\Omega_{c}$ of $u$ for $c \in(0,1)$ and the support of $u$ have Lipschitzcontinuous boundary.

For the proof we can construct a nonempty cone $C$ with vertex on $\partial \Omega_{c}$ such that $\partial \Omega_{c} \cap C$ consists only of the vertex. Notice that (2.7) holds if $\Omega_{1}$ is convex.

EXAMPLE 3. An exterior boundary value problem arising in potential flow. Let $u: \mathbf{R}^{n} \rightarrow \mathbf{R}$ be a solution of the variational problem

$$
\begin{aligned}
\operatorname{Min}_{v \in A_{3}} J_{3}(v):=\int_{\mathbf{R}^{n} \backslash \Omega_{1}}\left\{|\nabla v(x)|^{2}+\lambda^{2} \chi_{\{v>0\}}(x)\right\} d x \\
\text { where } A_{3}:=\left\{v \in W_{\text {loc }}^{1,2}\left(\mathbf{R}^{n}\right) \mid v \equiv 1 \text { on } \bar{\Omega}_{1}\right\} .
\end{aligned}
$$


Here $\chi_{S}$ denotes the characteristic function of a set $S$. The following results were derived in the pioneering paper [1] of H. W. Alt and L. Caffarelli. Let $\partial \Omega_{1}$ be sufficiently smooth and $\Omega_{1}$ bounded. Then there exists a solution $u \in A_{3}$ to problem (V3). Any solution of (V3) has bounded support and belongs to $C^{0,1}\left(\mathbf{R}^{n}\right)$. Furthermore, $u$ is harmonic in the set $\left\{x \in \mathbf{R}^{n} \backslash \bar{\Omega}_{1} \mid u(x)>0\right\}$, and $0 \leq u(x) \leq 1$ in $\mathbf{R}^{n}$. Formally $u$ is a solution to the free boundary problem

$$
\begin{aligned}
\Delta u=0 & \text { on }\{u>0\} \backslash \bar{\Omega}_{1}, \\
u=1 & \text { on } \partial \Omega_{1}, \\
u=0 & \text { and }|\partial u / \partial n|=\lambda \quad \text { on } \partial\{u>0\} .
\end{aligned}
$$

Notice that in particular the last condition has to be interpreted in a generalized sense, since the free boundary $\partial\{u>0\}$ might not be sufficiently smooth to define a normal vector field on it. Assuming a certain flatness condition, the free boundary is known to be of class $C^{1, \alpha}$ and even analytic. This flatness condition can be verified for the plane case $n=2$. If the free boundary satisfies the interior sphere condition, one can easily prove uniqueness $[\mathbf{2 7}, \mathbf{2 8}$; ; see also [25].

For dimensions $n \geq 3$ one has examples of solutions to (2.8), (2.9), (2.10) with singular free boundary.

COROLlaRY 2.4. Suppose that $u$ is any solution of problem (V4), that $\Omega_{1}$ is bounded and satisfies (2.7). Then all the level sets of $u$ are starshaped and the free boundary $\partial\{u>0\}$ is Lipschitz continuous.

For the proof we can modify an idea in $[9, \S 1]$ and show that different solutions $u$ and $v$ of (V4) are nested in the sense of Corollary 2.1(iii). Then we can proceed as in the proof of Corollary 2.1.

In two dimensions the starshapedness of $\{u>0\}$ was derived in [28] by a different method.

An extension of this application to functionals of type

$$
J(v)=\int_{\mathbf{R}^{n} \backslash \bar{\Omega}_{1}}\left\{|\nabla v(x)|^{2}+v^{\alpha}(x) \chi_{\{v>0\}}(x)\right\} d x, \quad \alpha \in(0,1),
$$

is possible. Such a functional was studied in [21].

EXAMPLE 4. An obstacle problem. Let $\Omega_{0} \subset \mathbf{R}^{n}$ be starshaped with respect to zero and let $\partial \Omega_{0}$ be sufficiently smooth. Let $\psi \in C^{1,1}\left(\bar{\Omega}_{0}\right)$ be given with $\psi<0$ on $\partial \Omega_{0}, \psi(0)=\max \left\{\psi(x) \mid x \in \bar{\Omega}_{0}\right\}$, and suppose that all the level sets of $\psi$ are starshaped with respect to zero. Let $u$ be a solution of the variational problem

$$
\operatorname{Min}_{v \in A_{4}} J_{1}(v), \quad \text { where } A_{4}:=\left\{v \in W^{1,2}\left(\Omega_{0}\right) \mid v \geq \psi \text { a.e. in } \Omega_{0}\right\} .
$$

Then under the assumptions of Corollary 2.1 the function $u$ has starshaped level sets. This can be deduced by cutting off $\psi$ at "height" $\psi(0)-\delta$ and by the limiting process $\delta \rightarrow 0^{+}[\mathbf{1 3}]$. For another proof under the stronger assumptions $x \cdot \nabla \psi<0$ in $\Omega_{0} \backslash\{0\}$ and $F$ convex, we refer to $[\mathbf{1 1}]$.

\section{REFERENCES}

1. H. W. Alt and L. A. Caffarelli, Existence and regularity for a minimum problem with free boundary, J. Reine Angew. Math. 325 (1981), 105-144.

2. C. Bandle and M. Marcus, Radial averaging transformation with various metrics, Pacific J. Math. 46 (1973), 337-348. 
3. __ Radial averaging transformations and generalized capacities, Math. Z. 145 (1975), $11-17$.

4. J. I. Diaz and M. A. Herrero, Estimate on the support of the solutions of some nonlinear elliptic and parabolic problems, Proc. Roy. Soc. Edinburgh Sect. A 89 (1981), 347-359.

5. H. Federer, Curvature measure, Trans. Amer. Math. Soc. 93 (1959), 418-491.

6. L. E. Fraenkel and M. S. Berger, A global theory of steady vortex rings in an ideal fluid, Acta Math. 132 (1974), 13-51.

7. L. S. Frank and W. D. Wendt, On an elliptic operator with discontinuous nonlinearity, J. Differential Equations 54 (1984), 1-18.

8. A. Friedman, Variational principles and free boundary problems, Wiley, New York, 1982.

9. A. Friedman and D. Phillips, The free boundary of a semilinear elliptic equation, Trans. Amer. Math. Soc. 282 (1984), 153-182.

10. H. Grabmüller, $A$ note on equimeasurable starshaped rearrangement or: "How long is John Miller's nose?", Report 096, Erlangen, 1983.

11. B. Kawohl, Starshapedness of level sets for the obstacle problem and for the capacitory potential problem, Proc. Amer. Math. Soc. 89 (1983), 637-640.

12. __ A geometric property of level sets of solutions to semilinear elliptic Dirichlet problems, Appl. Anal. 16 (1983), 229-234.

13. _ Geometrical properties of level sets of solutions to elliptic problems, Proc. Sympos. Pure Math., vol. 45, part 2, Amer. Math. Soc., Providence, R.I., 1986, pp. 25-36.

14. __ Starshaped rearrangement and applications, LCDS Report 83-20, Brown Univ., Providence, R.I., 1983.

15. M. Marcus, Transformations of domains in the plane and applications in the theory of functions. Pacific J. Math. 14 (1964), 613-626.

16. __ Radial averaging of domains, estimates for Dirichlet integrals and applications, J. Analyse Math. 27 (1974), 47-93.

17. D. S. Mitrinović, Analytic inequalities, Springer, Berlin, 1970.

18. J. Neðas, Les méthodes directes en théorie des équations elliptiques, Academia, Prague, 1967.

19. L. E. Payne, On two conjectures in the fixed membrane eigenvalue problem, Z. Angew. Math. Phys. 24 (1973), 721-729.

20. L. E. Payne and A. Weinstein, Capacity, virtual mass, and generalized symmetrization, Pacific J. Math. 2 (1952), 633-641.

21. D. Phillips, A minimization problem and the regularity of solutions in the presence of a free boundary, Indiana Univ. Math. J. 32 (1983), 1-17.

22. J. A. Pfaltzgraff, Radial symmetrization and capacities in space, Duke Math. J. 34 (1967), 747-756.

23. G Polya and G. Szegö, Isoperimetric inequalities in mathematical physics, Ann. Math. Stud., no. 27, Princeton Univ. Press, Princeton, N.J., 1951.

24. R. Sperb, Extension of two theorems of Payne to some nonlinear Dirichlet problem, $\mathrm{Z}$. Angew. Math. Phys. 26 (1975), 721-726.

25. J. Spruck, Uniqueness in a diffusion model of population biology, Comm. Partial Differential Equations 8 (1983), 1605-1620.

26. G. Szegö, On a certain kind of symmetrization and its applications, Ann. Mat. Pura Appl. (4) 40 (1955), 113-119.

27. D. F. Tepper, Free boundary problem, SIAM J. Math. Anal. 5 (1974), 841-846.

28. - On a free boundary problem, the starlike case, SIAM J. Math. Anal. 6 (1975), 503-505.

SFB 123, Universität Heidelberg, Im Neuenheimer feld 294, D 6900 HeidelBERG, FEDERAL REPUblic OF GERMANY 